\title{
Determination of the Burning Velocity Domain of a Statistically Stationary Turbulent Premixed Flame in Presence of Counter-Gradient Transport
}

\author{
V. A. Sabel'nikov' ${ }^{1}$ and P. Bruel ${ }^{2}$ \\ ${ }^{1}$ Onera, The French Aerospace Lab, F-91761 Palaiseau, France \\ ${ }^{2}$ Centre National de la Recherche Scientifique, Laboratoire de Mathématiques et de leurs Applications de Pau, \\ UMR 5142 CNRS-Université de Pau et des Pays de l'Adour, IPRA-BP 115564013 PAU Cedex, France
}

Correspondence should be addressed to P. Bruel, pascal.bruel@univ-pau.fr

Received 15 February 2011; Accepted 15 April 2011

Academic Editor: Andrei N. Lipatnikov

Copyright ( 2011 V. A. Sabel'nikov and P. Bruel. This is an open access article distributed under the Creative Commons Attribution License, which permits unrestricted use, distribution, and reproduction in any medium, provided the original work is properly cited.

\begin{abstract}
The present study aims at providing a complete picture of the various propagation scenarios that a statistically stationary turbulent premixed flame may possibly undergo. By explicitly splitting the scalar turbulent flux between its gradient and counter-gradient contributions, the scalar governing equation is rewritten as an ordinary differential equation in the phase space. Then, an analysis of the characteristic equations in the vicinity of the reactants and products side is carried out. The domain of existence of the propagation velocity is then determined and positioned over the relevant Bray number range. It is shown in particular that when a counter-gradient transport at the cold leading edge of the flame is dominant, there still exists a possibility of observing a steady regime of propagation. This conclusion is compatible with recent experimental data and observations based on the analysis of direct numerical simulations.
\end{abstract}

\section{Introduction}

The determination of the burning velocity of turbulent premixed flames $S_{T}$ has been the subject of many experimental studies. As far as a given modelling of such reacting flows is concerned, the a priori value of $S_{T}$ for statistically stationary one-dimensional turbulent flame is often estimated through the direct application of the results of the KolmogorovPetrovskii-Piskounov (KPP) theory [1] which showed that, for a one-dimensional reaction zone without heat release described through the evolution of a single progress variable $c$ and with a constant diffusion coefficient $D_{t}$, the steady propagation of the reaction zone is controlled by the behavior of the reaction rate at the reactants side and by the value of the diffusion coefficient. In many situations, experiments (Moss [2], Cheng and Shepherd [3], Troiani et al. [4], Zimmer et al. [5]) as well as direct numerical simulations (DNS) (Veynante et al. [6], Nishiki [7], Hauguel [8], Lee and Huh [9]) show that an expression of the turbulent transports via a gradient expression is not appropriate as some mechanisms may promote a transport with a sign identical to that of the mean scalar gradient. An extensive review of relevant experimental and DNS data can be found in the paper of Lipatnikov and Chomiak [10]. In such situations, the introduction of the Bray number proposed by Veynante et al. [6] proved to be helpful to discriminate between flow configurations featuring gradient turbulent flux (GTF) only $\left(N_{B} \leq 1\right)$ or counter-gradient turbulent flux (CGTF) $\left(N_{B} \geq 1\right)$. The Bray number was defined as $N_{B}=\tau S_{L} / 2 \alpha_{v} u^{\prime}$ where $\tau$ designates the heat release parameter and $\alpha_{v}$ some order unity efficiency function which depends mainly on (and is an increasing function of) the ratio between the turbulence integral length scale and the flame thermal thickness. For $N_{B} \geq 1$, one of the most prominent mechanism leading to CGTF is directly related to the differential effect of the pressure gradient on the pockets of reactants (heavy) and products (lighter). But it should, nevertheless, be stressed, that even when such a mechanism 
is predominant, there still exists a gradient turbulent flux acting at scales much smaller than those of the pockets and for which the turbulent transport coefficient cannot be estimated through a turbulence model which integrates the whole turbulence scales. Accordingly, and following in that respect Veynante et al. [6], Zimont and Biagioli [11] or Lipatnikov and Chomiak [12], this suggests to consider the turbulent scalar flux as resulting from the competition between gradient and counter-gradient mechanisms.

Along these lines, we propose to study the propagation properties of a turbulent premixed flame by considering such a flux decomposition. First, an extended KPP analysis is developed. It combines all the possible scenarios with the change of $N_{B}$ and provides a general analysis whose results are not qualitatively dependent anymore on the modelling closures chosen but permit a clear and comprehensive discrimination between all the various propagation regimes.

We note that, in the framework of an eddy-breakup model, an analytical attempt aimed at explaining the influence of the presence of CGTF on the turbulent flame propagation properties has been done by Corvellec et al. [13] who considered an idealized one-dimensional premixed flame that propagates through high-Reynoldsnumber frozen turbulence. These authors dealt only with a flame brush formed by a GTF zone followed by a CGTF one. The scalar flux in each zone was expressed by employing a classical gradient formulation with the peculiarity of using a strictly negative diffusion coefficient for the CGTF zone. Thanks to this mathematical "trick", it was then possible to employ the KPP technique to study the characteristics of the governing equation at both sides of the flame brush and at the intermediate point at which the flux is zero. This approach did not put into evidence a qualitative change in the nature of the burning velocity domain which appears to be still a semi-infinite interval. The lower bound though appeared to be controlled by both sides in such a situation. It drew also the attention on the fact that the KPP results cannot be extrapolated directly in such situations (in accordance to some extent with the numerical simulations of Bradley et al. [14]). The results of Corvellec et al. [13] cannot be considered as being of general implication since they are contingent to the recourse to an effective diffusion coefficient and are limited to the analysis of only one flame structure, that is, a GTF zone followed by a CGTF one. The Bray number is absent in the theory developed in [13] whereas DNS $[6,9]$ and recent experiments on turbulent premixed flames $[4,5]$ show that a different mean flame structure may be encountered that is, a CGTF zone followed by a GTF one. Thus, the present approach is more general than those followed previously since it permits to cover all the two-zone mean flame structures that can be thought of. It shows in particular that as far as the burning velocity domain is concerned, qualitative differences with the KPP and Corvellec et al. [13] results are obtained.

\section{Mathematical Formulation}

2.1. Governing Equations. An unsteady 1-D freely developing turbulent isenthalpic premixed flame is considered here and is described through the evolution of a single progress variable $c=\left(T-T_{r}\right) /\left(T_{b}-T_{r}\right)$, where subscripts $r$ and $b$ denote the states of the reactant mixture and the fully burnt products, respectively, and $\tau=\left(T_{b}-T_{r}\right) / T_{r}$ defines the heat release parameter. Thus, in a fixed coordinate system $(O, x)$ the Favre averaged equation for $c$ and the continuity equation to be used later on, are given by

$$
\begin{gathered}
\frac{\partial \bar{\rho}}{\partial t}+\frac{\partial \bar{\rho} \tilde{u}}{\partial x}=0, \\
\frac{\partial \bar{\rho} \tilde{c}}{\partial t}+\frac{\partial \bar{\rho} \tilde{u} \tilde{c}}{\partial x}=-\frac{\partial}{\partial x} \overline{\rho u^{\prime \prime} c^{\prime \prime}}+\bar{w}
\end{gathered}
$$

$\overline{\rho u^{\prime \prime} c^{\prime \prime}}$ is the turbulent flux, and $\bar{w}$ is the mean reaction rate whose exact expression is not required at this stage. The flame is propagating from right to left, that is, $\partial \widetilde{c} / \partial x \geqslant 0$.

We consider here the case for which counter-gradient mechanism is largely present in the flame as it is the case when the turbulence intensity is sufficiently low and/or the heat release parameter sufficiently large (see [6] for further details). Accordingly, the turbulent flux is expressed as

$$
F=\overline{\rho u^{\prime \prime} c^{\prime \prime}}=F_{\mathrm{GTF}}+F_{\mathrm{CGTF}}
$$

$F_{\text {GTF }}<0$ represents the gradient contribution while $F_{\text {CGTF }}>0$ corresponds to the counter-gradient part. Such a decomposition is compatible with the findings of Veynante et al. [6] who derived an algebraic expression for the flux as a function of the laminar flame velocity $S_{L}$, the heat release parameter $\tau$ and the rms velocity $u^{\prime}$, namely, $\overline{\rho u^{\prime \prime} c^{\prime \prime}}=\bar{\rho} \widetilde{c}(1-$ $\widetilde{c})\left(\tau S_{L}-2 \alpha_{\nu} u^{\prime}\right)$. In this particular case, the counter-gradient contribution is equal to

$$
F_{\mathrm{CGTF}}=\bar{\rho} \widetilde{c}(1-\tilde{c}) \tau S_{L}
$$

and the gradient one reads as

$$
F_{\mathrm{GTF}}=-2 \alpha_{v} u^{\prime} \bar{\rho} \widetilde{c}(1-\widetilde{c}) .
$$

The ratio $F_{\mathrm{CGTF}} /\left|F_{\mathrm{GTF}}\right|$ is just equivalent to the Bray number $N_{B}$. If we introduce $D_{t} \approx l_{t} u^{\prime}$, where $l_{t}$ is the turbulence integral length scale and suppose that $\partial \widetilde{c} / \partial x \approx$ $\tilde{c}(1-\tilde{c}) / l_{t}$, then the gradient contribution can be classically written as $F_{\mathrm{GTF}}=-\bar{\rho} D_{t} \partial \tilde{c} / \partial x$. Here, the dependency of $\alpha_{v}$ on the ratio between the flame thermal thickness and the turbulence integral length scale has been neglected. In the following analysis, we shall consider $F_{\mathrm{GTF}}$ as given by (4) combined with a more general expression for $F_{\mathrm{CGTF}}$, namely,

$$
F_{\mathrm{CGTF}}=\bar{\rho} \tau S_{L} f(\tilde{c}),
$$

where $f$ is a positive continuous function of $\tilde{c}$ such that $f(0)=f(1)=0$. In the case of Veynante et al. [6], $f(\widetilde{c})$ is a quadratic symmetric function, namely,

$$
f(\widetilde{c})=\widetilde{c}(1-\tilde{c})
$$

If we now rewrite the $\tilde{c}$-equation combined with the continuity equation, one obtains

$$
\bar{\rho} \frac{\partial \tilde{c}}{\partial t}+\left(\bar{\rho} \tilde{u}+\tau S_{L} \frac{\partial}{\partial \tilde{c}} \bar{\rho} f(\tilde{c})\right) \frac{\partial \widetilde{c}}{\partial x}=\frac{\partial}{\partial x}\left(\bar{\rho} D_{t} \frac{\partial \tilde{c}}{\partial x}\right)+\bar{w} .
$$


It is worth noting that the analysis could be done also in the case, not considered here, where $F$ would be expressed without the introduction of a turbulent diffusion coefficient (e.g., using directly the decomposition of Veynante et al. [6]).

We rewrite (7) in a nondimensional form by defining the following reference parameters:

(1) a velocity scale $\hat{u}=u^{\prime}$,

(2) a time scale $\hat{t}=l_{t} / u^{\prime}$,

(3) a length scale $\hat{l}$ proportional to the integral turbulence scale, that is, $\hat{l}=l_{t}$,

(4) a reference turbulent diffusion coefficient $D_{t_{0}}=l_{t} u^{\prime}$ ( $l_{t}$ is chosen in such a way that the proportionality coefficient between $D_{t_{0}}$ and $l_{t} u^{\prime}$ is unity),

(5) a flame velocity scale $\sqrt{D_{t_{0}} / \hat{t}}=u^{\prime}$.

Thus, one has

$$
\begin{aligned}
R \frac{\partial \widetilde{c}}{\partial t^{*}} & +\left(R u^{*}+N_{B} 2 \alpha_{v} \frac{\partial}{\partial \widetilde{c}}(R f)\right) \frac{\partial \tilde{c}}{\partial x^{*}} \\
& =\frac{\partial}{\partial x^{*}}\left(R D \frac{\partial \tilde{c}}{\partial x^{*}}\right)+\bar{w}^{*},
\end{aligned}
$$

where $x^{*}=x / \hat{l}, u^{*}=\tilde{u} / \hat{u}, t^{*}=t / \hat{t}, \mathbb{D}=D_{t} / D_{t_{0}}, R=\bar{\rho} / \rho_{u}=$ $1 / 1+\tau \widetilde{\mathcal{c}}$, and $\bar{w}^{*}=\bar{w}\left(\hat{t} / \rho_{u}\right)$. In the following and whenever unambiguous, we shall drop superscript ${ }^{*}$. So, with that convention, (8) reads as

$$
R \frac{\partial \tilde{c}}{\partial t}+\left(R u+N_{B} 2 \alpha_{v} \frac{\partial}{\partial \widetilde{c}}(R f)\right) \frac{\partial \tilde{c}}{\partial x}=\frac{\partial}{\partial x}\left(R D \frac{\partial \widetilde{c}}{\partial x}\right)+\bar{w},
$$

and the nondimensional form of the continuity equation (1) is given by

$$
\frac{\partial R}{\partial t}+\frac{\partial R u}{\partial x}=0
$$

At this stage, it is important to underline that the form of (7) allows us to give a physical interpretation of the influence of CGTF on the flame structure (following mainly the reasoning adopted in [6]). The term of CGTF is a nonlinear convection term which tends to move both sides toward each other because of the change of sign of $\partial \bar{\rho} f(\widetilde{c}) / \partial \widetilde{c}$ (positive at the fresh reactants side and then negative at the burnt products side). Such a "thinning" effect has to be counter-balanced by the gradient transport term in order to obtain a steady state flame propagation regime.

2.2. Steady-State Regime of Flame Propagation. Let us consider a steady state regime of flame propagation. Accordingly, in a coordinate system attached to the mean flame brush and introducing the flame mass consumption $\dot{m}$ and the turbulent flame speed $S_{t}$ such as $\dot{m}=\rho_{u} S_{t}$, the preceding continuity and progress-variable nondimensional equations are written as

$$
\begin{gathered}
R u=\frac{\dot{m}}{\rho_{u} \mathcal{u}^{\prime}}=\frac{\rho_{u} S_{t}}{\rho_{u} \mathcal{u}^{\prime}}=\frac{\rho_{b} u_{b}}{\rho_{u} \mathcal{u}^{\prime}}=\Lambda, \\
{\left[\Lambda+N_{B} 2 \alpha_{v} \frac{d}{d \widetilde{c}}(R f)\right] \frac{d \tilde{c}}{d x}=\frac{d}{d x}\left(R D \frac{d \widetilde{c}}{d x}\right)+\bar{w}}
\end{gathered}
$$

where $\Lambda=S_{t} / u^{\prime}$.

We can rewrite (11) as a first-order differential equation for $P=(R \mathscr{D} / \Lambda)(d \tilde{c} / d x)$ on the $\tilde{c}$-domain $[0,1]$, namely

$$
\left[1+\frac{N_{B} 2 \alpha_{v}}{\Lambda} \frac{d}{d \widetilde{c}}(R f)\right] P=P P^{\prime}+\bar{w} \frac{R D}{\Lambda^{2}} .
$$

Introducing $\hat{N}_{B}=N_{B} 2 \alpha_{v}$ and $\Omega=\bar{w} R \mathscr{D},(12)$ is expressed as

$$
P P^{\prime}=\left[1+\frac{\hat{N}_{B}}{\Lambda}(R f)^{\prime}\right] P-\Omega \frac{1}{\Lambda^{2}},
$$

where ()$^{\prime}$ denotes the derivation with respect to $\tilde{c}$.

\section{Analysis of the $P$-Equation in the Vicinity of the Singular Points}

Now, our primary objective is to determine the domain of existence of $\Lambda$. In principle, we need for that to analyse the trajectories in the $(P, \widetilde{c})$ phase plane in the KPP-like manner. It is clear that a priori, due to the presence of the CGTF derivative $f^{\prime}$, it is difficult to perform the KPP analysis in such a case.

Thus, leaving aside the question of determining the trajectories themselves, we focus on the determination of the associated $\Lambda$ domain by examining the characteristic equations of (13) at both sides of the brush. The implication of the relative position of $P$ and $F=\left(\hat{N}_{B} / \Lambda\right) R f$ for the $P$-trajectories when they are tangent to the characteristics lines is also analyzed. We denote $F_{0}^{\prime}=d F(0) / d \tilde{c}=$ $\hat{N}_{B} / \Lambda(R f)^{\prime}(0)=\left(\hat{N}_{B} / \Lambda\right) f^{\prime}(0)=\alpha$ and $F_{1}^{\prime}=-d F(1) / d \widetilde{c}=$ $-\hat{N}_{B} / \Lambda(R f)^{\prime}(1)=-(1 /(\tau+1))\left(\hat{N}_{B} / \Lambda\right) f^{\prime}(1)=\beta=(1 /(\tau+$ 1)) $\alpha\left|f^{\prime}(1)\right| / f^{\prime}(0)$ and introduce the two functions $f_{1}(\alpha)=$ $4 /(1+\alpha)^{2}$ and $f_{2}(\alpha)=1 / \alpha$, such that $f_{2}(\alpha)>f_{1}(\alpha)$ for all $\alpha$ but $\alpha=1$ for which $f_{2}(1)=f_{1}(1)$.

(a) Reactants Side. In the limit $\tilde{c} \rightarrow 0^{+}$and assuming that $P=s \tilde{c}$ and $F=\alpha \tilde{c}(s>0$ and $\alpha>0)$, the characteristic equation associated with (13) is given by

$$
s^{2}-s(1+\alpha)+\frac{1}{\Lambda^{2}} \Omega^{\prime}(0)=0 .
$$

The required positiveness of the discriminant $\Delta_{0^{+}}=(1+$ $\alpha)^{2}-\left(4 / \Lambda^{2}\right) \Omega^{\prime}(0)$ is assured as soon as $\Lambda^{2}>f_{1}(\alpha) \Omega^{\prime}(0)$. The corresponding roots $s_{1}=(1+\alpha) / 2+\sqrt{\Delta_{0^{+}} / 4}$ and $s_{2}=$ $(1+\alpha) / 2-\sqrt{\Delta_{0^{+}} / 4}$ are both positive with $s_{1}>s_{2}$. Accordingly, the reactants side is an improper unstable node and all 
the trajectories but one (the characteristic line $s_{1} \tilde{c}$ itself) are tangent to the $s_{2} \tilde{c}$ line. Two cases are to be considered in the vicinity of $\tilde{c}=0$ : (1) $F_{\mathrm{GTF}}+F_{\mathrm{CGTF}}<0$ (the gradient contribution overcomes the counter-gradient one) and (2) the reverse situation prevails, that is, $F_{\mathrm{GTF}}+F_{\mathrm{CGTF}}>0$.

(1) Gradient transport prevails, that is, $F_{\mathrm{GTF}}+F_{\mathrm{CGTF}}<$ $0 \Leftrightarrow P>F$. This implies that $s_{2}>\alpha$. Solving this inequality yields $\alpha<1$ and $\Lambda^{2}<f_{2} \Omega^{\prime}(0)$. It is worth noting that when $\alpha \rightarrow 0$, one recovers the KPP domain.

(2) Counter-gradient transport prevails, that is, $F_{\mathrm{GTF}}+$ $F_{\text {CGTF }}>0 \Leftrightarrow P<F$. Two scenarios meet these inequalities, namely.

(i) $\alpha>s_{1}$. Solving this inequality yields $\alpha>1$ and $\Lambda^{2}<$ $f_{2} \Omega^{\prime}(0)$.

(ii) $s_{2}<\alpha<s_{1}$. This implies that $\Lambda^{2}>f_{2} \Omega^{\prime}(0)$ for $\alpha>0$.

In both cases $(1,2)$, we must satisfy also

$$
\Lambda^{2}>f_{1} \Omega^{\prime}(0) .
$$

Thus, for case 1 we have

$$
f_{1} \Omega^{\prime}(0)<\Lambda^{2}<f_{2} \Omega^{\prime}(0), \quad \alpha<1,
$$

and for case 2

$$
\begin{gathered}
f_{1} \Omega^{\prime}(0)<\Lambda^{2}<f_{2} \Omega^{\prime}(0), \quad \alpha>1, \\
\Lambda^{2}>f_{2} \Omega^{\prime}(0), \quad \alpha>0 .
\end{gathered}
$$

Using the definitions of $f_{1}(\alpha)=4 /(1+\alpha)^{2}, f_{2}(\alpha)=1 / \alpha$ and $\alpha=\left(\widehat{N}_{B} / \Lambda\right) f^{\prime}(0)$, for case 1 , one has

$$
2 \sqrt{\Omega^{\prime}(0)}-\hat{N}_{B} f^{\prime}(0)<\Lambda<\frac{\Omega^{\prime}(0)}{\widehat{N}_{B} f^{\prime}(0)}, \quad \Lambda>\widehat{N}_{B} f^{\prime}(0)
$$

or in equivalent form

$$
2 \sqrt{\Omega^{\prime}(0)}-\widehat{N}_{B} f^{\prime}(0)<\Lambda<\frac{\Omega^{\prime}(0)}{\hat{N}_{B} f^{\prime}(0)}, \quad \hat{N}_{B} f^{\prime}(0)<\sqrt{\Omega^{\prime}(0)} .
$$

For case 2, we have

$$
\begin{gathered}
2 \sqrt{\Omega^{\prime}(0)}-\widehat{N}_{B} f^{\prime}(0)<\Lambda<\frac{\Omega^{\prime}(0)}{\hat{N}_{B} f^{\prime}(0)}, \quad \hat{N}_{B} f^{\prime}(0)>\sqrt{\Omega^{\prime}(0)}, \\
\Lambda>\frac{\Omega^{\prime}(0)}{\hat{N}_{B} f^{\prime}(0)}, \quad \hat{N}_{B}>0 .
\end{gathered}
$$

Introducing the variables

$$
\tilde{\Lambda}=\frac{\Lambda}{\sqrt{\Omega^{\prime}(0)}}, \quad \tilde{N}_{B}=\frac{\hat{N}_{B} f^{\prime}(0)}{\sqrt{\Omega^{\prime}(0)}},
$$

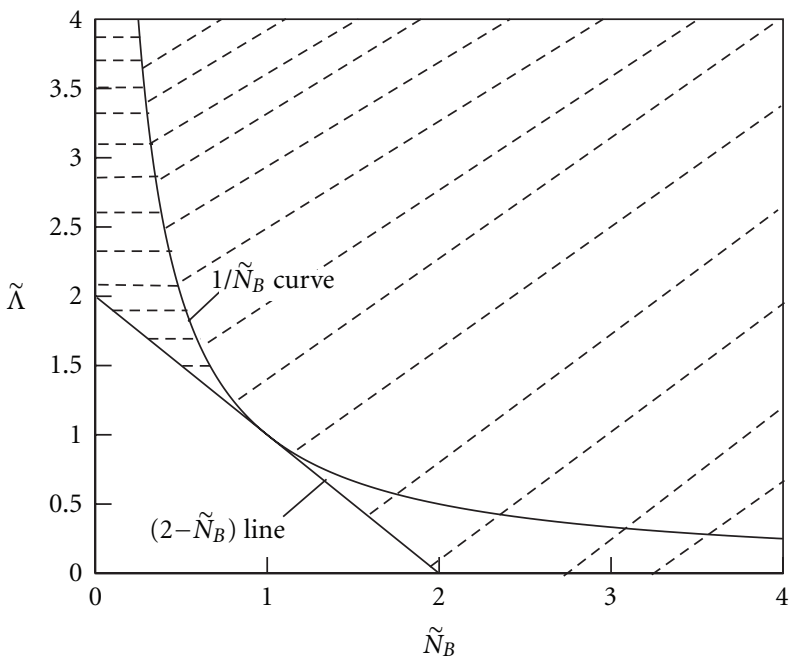

E三 GTF

ए; CGTP

FIGURE 1: Domain of existence in the $\left(\tilde{\Lambda}, \tilde{N}_{B}\right)$ plane of gradient or counter-gradient total turbulent flux at the reactants side.

we can rewrite for case 1

$$
2-\tilde{N}_{B}<\tilde{\Lambda}<\frac{1}{\widetilde{N}_{B}}, \quad \tilde{N}_{B}<1,
$$

and for case 2

$$
\begin{gathered}
2-\tilde{N}_{B}<\tilde{\Lambda}<\frac{1}{\widetilde{N}_{B}}, \quad \tilde{N}_{B}>1, \\
\tilde{\Lambda}>\frac{1}{\widetilde{N}_{B}}, \quad \tilde{N}_{B}>0 .
\end{gathered}
$$

It is important to note that relations in (23) are the sum of two sets, but not the product. Figure 1 illustrates the domain of existence of GTF and CGTF in the plane $\left(\widetilde{\Lambda}, \tilde{N}_{B}\right)$ that followed from the analysis of (13) (for $P$ ) in the vicinity $\tilde{c} \rightarrow 0$.

(b) Products Side. In the limit $\tilde{c} \rightarrow 1^{-}$and assuming that $P=s(1-\tilde{c})$ and $F=\beta(1-\tilde{c})(s>0$ and $\beta>0)$, the characteristic equation associated with (13) is given by

$$
s^{2}+s(1-\beta)+\frac{1}{\Lambda^{2}} \Omega_{\tilde{c}}^{\prime}(1)=0 .
$$

The discriminant $\Delta_{1^{-}}$is always positive and there exists only one positive root $s_{3}=(\beta-1) / 2+\sqrt{\Delta_{1-} / 4}$. Accordingly, the products side is a saddle and all the trajectories are tangent to the $s_{3} \tilde{c}$ line. Again, two situations are to be considered:

(1) GTF prevails, that is, $F_{\mathrm{GTF}}+F_{\mathrm{CGTF}}<0 \Leftrightarrow P>F$. This implies that $\beta<s_{3}$ or equivalently $\Lambda^{2}<(1 / \beta)\left|\Omega^{\prime}(1)\right|$. In the limit $\beta \rightarrow 0$, the original KPP behavior is recovered, that is, the absence of constraint. 


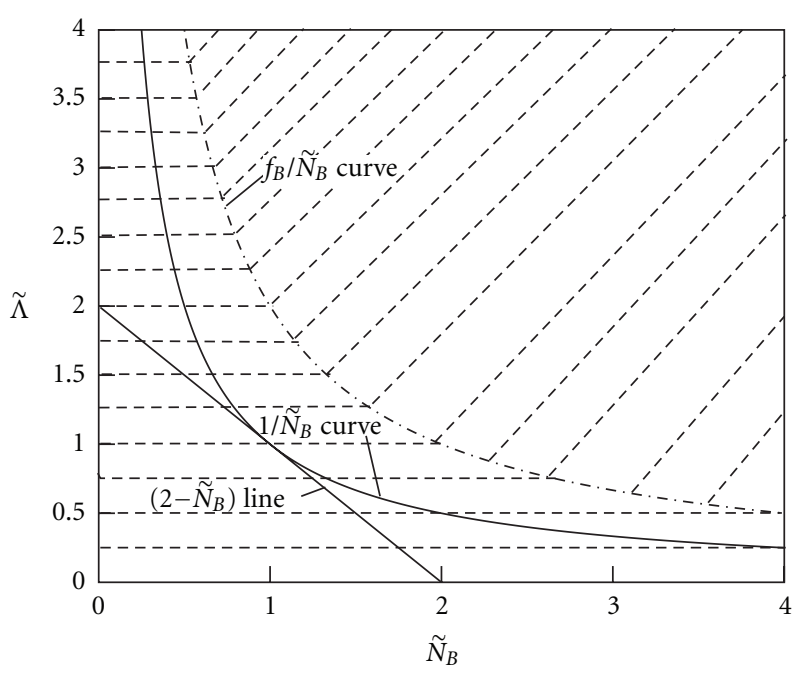

E三ョ GTF

E's CGTF

(a)

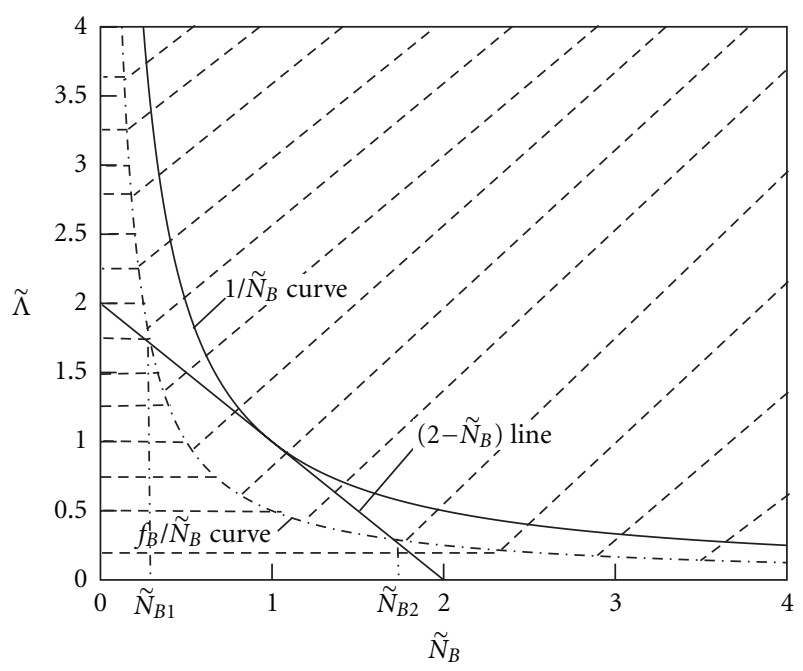

E三 GTF

' CGTF

(b)

FIGURE 2: Domain of existence in the $\left(\tilde{\Lambda}, \tilde{N}_{B}\right)$ plane of gradient or counter-gradient total turbulent flux at the products side (a) case $f_{B}>1$ and (b) case $f_{B}<1$.

(2) CGTF prevails, that is, $F_{\mathrm{GTF}}+F_{\mathrm{CGTF}}>0 \Longleftrightarrow P<$ $F$. This implies that $\beta>s_{3}$ or equivalently $\Lambda^{2}>$ $(1 / \beta)\left|\Omega^{\prime}(1)\right|$.

Using the relation

$$
\frac{\beta}{\alpha}=\frac{1}{1+\tau} \frac{\left|f^{\prime}(1)\right|}{f^{\prime}(0)}
$$

we transform the above inequalities into the following form:
Table 1

\begin{tabular}{lcccc}
\hline Configuration & G/G & G/CG & CG/G & CG/CG \\
Reactants side & G & G & CG & CG \\
Products side & G & CG & G & CG \\
\hline
\end{tabular}

for case 1 (GTF)

$$
\widetilde{\Lambda}<\frac{1}{\tilde{N}_{B}} f_{B}
$$

for case 2 (CGTF)

$$
\tilde{\Lambda}>\frac{1}{\tilde{N}_{B}} f_{B}
$$

where

$$
f_{B}=(\tau+1) \frac{f^{\prime}(0)}{\left|f^{\prime}(1)\right|} \frac{\left|\Omega^{\prime}(1)\right|}{\Omega^{\prime}(0)} .
$$

Figures 2(a) and 2(b) present the domain of GTF and CGTF obtained from the analysis in the vicinity $\tilde{c} \rightarrow 1$ and for $f_{B}>1$ and $f_{B}<1$, respectively. In the latter case, two remarkable values $\tilde{N}_{B_{1}}$ and $\tilde{N}_{B_{2}}$ of $\tilde{N}_{B}$ are introduced. They are defined by $\tilde{N}_{B_{1}}=\left(1-\sqrt{1-f_{B}}\right)<1$ and $\tilde{N}_{B_{2}}=\left(1+\sqrt{1-f_{B}}\right)>$ 1.

There exists a maximum of four possible configurations, listed in Table 1, which correspond to the total turbulent flux behavior (gradient (G) or counter-gradient (CG)) in the vicinity of the singular points $\tilde{c}=0$ and $\tilde{c}=1$.

Let us consider first the case $f_{B}>1$. Analysis of inequalities (22)-(23) and (25)-(27) gives the following results.

(i) G/G configuration:

$$
2-\widetilde{N_{B}}<\widetilde{\Lambda}<\frac{1}{\widetilde{N_{B}}}, \quad \widetilde{N_{B}}<1 .
$$

(ii) G/CG configuration is absent.

(iii) CG/G configuration:

$$
\left\{\begin{array}{c}
2-\widetilde{N_{B}}<\tilde{\Lambda}<\frac{1}{\widetilde{N}_{B}}, \widetilde{N_{B}}>1 \\
\widetilde{\Lambda}>\frac{1}{\widetilde{N_{B}}}, \widetilde{N_{B}}>0 \\
\tilde{\Lambda}<\frac{1}{\widetilde{N_{B}}} f_{B}, \widetilde{N_{B}}>0
\end{array}\right\} \text { sum of two sets }{ }_{\text {product. }}
$$

(iv) CG/CG Configuration:

$$
\left\{\begin{array}{c}
2-\widetilde{N_{B}}<\tilde{\Lambda}<\frac{1}{\widetilde{N}_{B}}, \widetilde{N_{B}}>1 \\
\widetilde{\Lambda}>\frac{1}{\widetilde{N}_{B}}, \widetilde{N_{B}}>0 \\
\tilde{\Lambda}>\frac{1}{\widetilde{N}_{B}} f_{B}, \widetilde{N_{B}}>0
\end{array}\right\} \text { sum of two sets } \text { product. }
$$




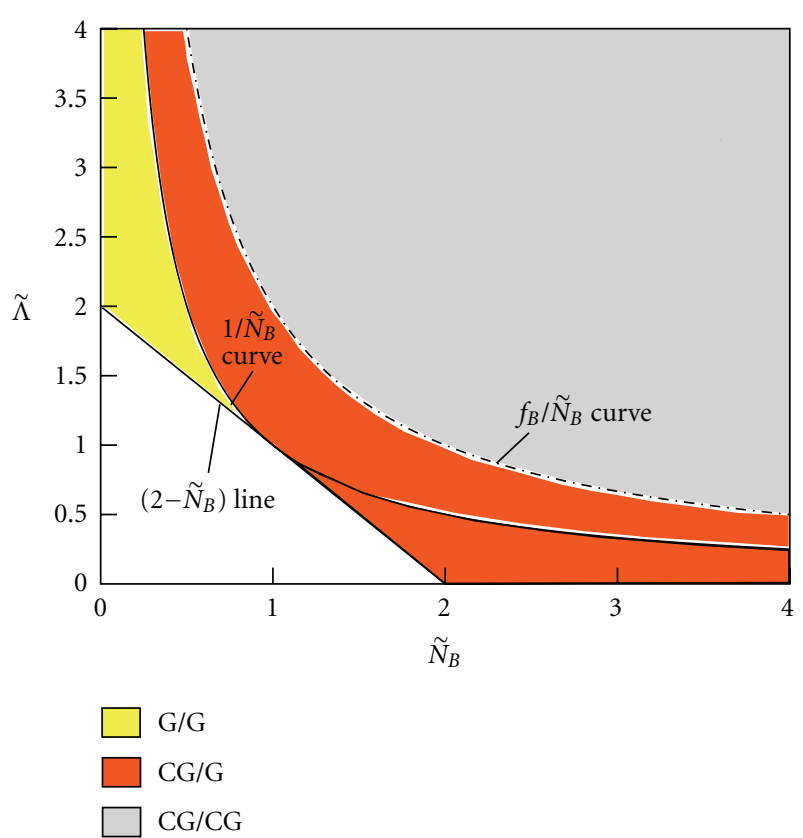

Figure 3: The domain of the three different possibilities for the turbulent flame brush structure in the plane $\left(\widetilde{\Lambda}, \tilde{N}_{B}\right)$ for $f_{B}>1$.

Domains of the above three possible configurations are given in Figure 3. Now for the case $f_{B}<1$, all four configurations are possible, namely,

(i) G/G configuration:

$$
\begin{aligned}
2- & \widetilde{N_{B}}<\widetilde{\Lambda}<\frac{f_{B}}{\widetilde{N_{B}}} \text { for } 0<\widetilde{N_{B}}<\widetilde{N_{B 1}} \text { with } \widetilde{N_{B 1}} \\
& =1-\sqrt{1-f_{B}}<1 .
\end{aligned}
$$

Consequently, in this interval $\left[0, \widetilde{N_{B 1}}\right]$, we have $\tilde{\Lambda}>1$.

(ii) G/CG configuration:

$f_{B} / \widetilde{N_{B}}<\tilde{\Lambda}<1 / \widetilde{N_{B}}$ for $0<\widetilde{N_{B}}<\widetilde{N_{B}}$ and $2-\widetilde{N_{B}}<\tilde{\Lambda}<$ $1 / \widetilde{N_{B}}$ for $\widetilde{N_{B}}<\widetilde{N_{B}}<1$ and again we have $\tilde{\Lambda}>1$.

(iii) $\mathrm{CG} / \mathrm{G}$ configuration:

$\max \left(0 ; 2-\widetilde{N_{B}}\right)<\tilde{\Lambda}<f_{B} / \widetilde{N_{B}}$ for $\widetilde{N_{B}}>\widetilde{N_{B 2}}$ with $\widetilde{N_{B 2}}=$ $1+\sqrt{1-f_{B}}>1$. In that case, we have $\tilde{\Lambda}<1$.

(iv) CG/CG configuration:

$$
\begin{gathered}
\tilde{\Lambda}>\frac{1}{\widetilde{N_{B}}} \quad \text { for } 0<\widetilde{N_{B}}<1, \\
\widetilde{\Lambda}>2-\widetilde{N_{B}} \quad \text { for } 1 \leq \widetilde{N_{B}}<\widetilde{N_{B 2}}, \\
\tilde{\Lambda}>\frac{f_{B}}{\widetilde{N_{B}}} \quad \text { for } \widetilde{N_{B}}>\widetilde{N_{B 2} .}
\end{gathered}
$$

The different regions of the $\left(\tilde{\Lambda}, \tilde{N}_{B}\right)$ plane corresponding to these four configurations are presented in Figure 4.

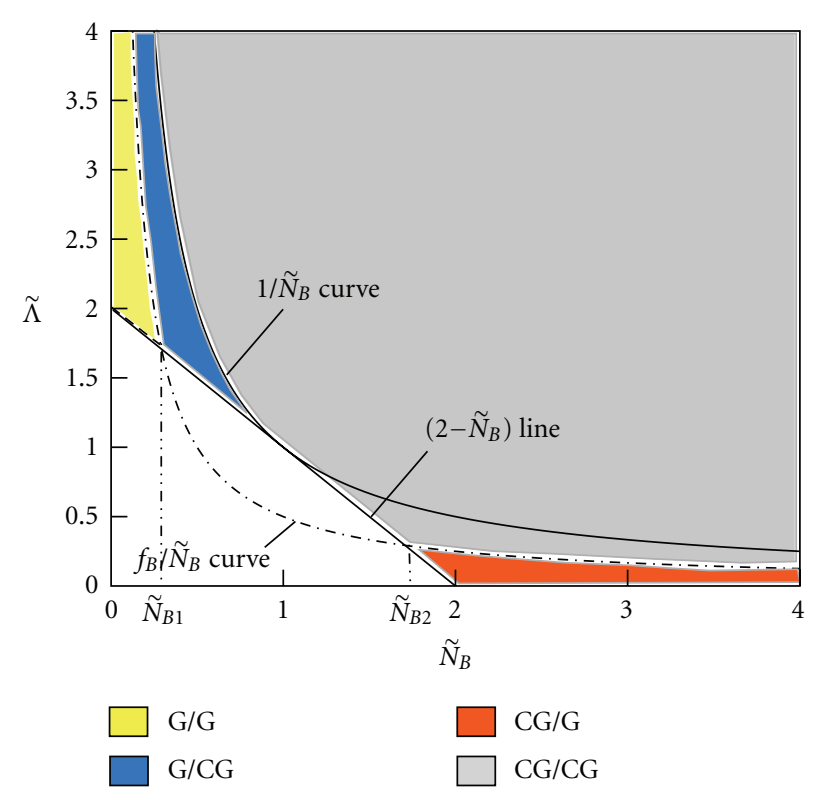

Figure 4: The domain of the three different possibilities for the turbulent flame brush structure in the plane $\left(\tilde{\Lambda}, \tilde{N}_{B}\right)$ for $f_{B}>1$.

We note that the case $f_{B}<1$ is realised for the classical eddybreak-up model where

$$
\bar{w}^{*}=\frac{\tilde{c}(1-\tilde{c})}{1+\tau \tilde{c}},
$$

and if $D_{t}=D_{t_{0}}=$ const, $f(\widetilde{c})=\widetilde{c}(1-\widetilde{c})$-Veynante et al. case [6]. In that case, one has $\Omega(\widetilde{c})=\widetilde{c}(1-\widetilde{c}) /(1+\tau \widetilde{c})^{2}$ and consequently $\Omega^{\prime}(0)=1$ and $\Omega^{\prime}(1)=-1 /(1+\tau)^{2}$. Thus,

$$
f_{B}=\frac{1}{1+\tau}<1
$$

The case $f_{B}>1$ is obtained if

$$
\bar{w}^{*}=\tilde{c}(1-\tilde{c})(1+\varkappa \widetilde{c}),
$$

where $\varkappa$ is some positive contant. So we have

$$
\begin{gathered}
\Omega(\tilde{c})=\frac{\tilde{c}(1-\tilde{c})(1+\varkappa \tilde{c})}{(1+\tau \tilde{\mathcal{c}})}, \\
\Omega^{\prime}(0)=1, \quad \Omega^{\prime}(1)=-\frac{1+\varkappa}{1+\tau} .
\end{gathered}
$$

For Veynante et al. [6], $f(\tilde{c})=\widetilde{c}(1-\tilde{c})$ and so

$$
f_{B}=(1+\tau) \frac{1+\varkappa}{1+\tau}=1+\varkappa>1 \quad \text { whenever } \varkappa>0 .
$$

It should be noted that experimental data $[3,15]$ suggest that $\bar{w}^{*}$ is shifted towards the burnt gas side. Such a situation can be modelled by relation (36) with $\varkappa>0$, and, in this case, we can have $f_{B}>1$. As was shown previously, in such a situation, the configuration G/CG is absent. Such a result is compatible with DNS results for 1-D freely propagating turbulent premixed flame $[6,9]$. It is also in correspondance 
with the recent experimental data of Troiani et al. [4] who investigated bluff-body stabilized turbulent premixed flames. These authors showed that the radial component of the scalar flux (which is the counterpart of $F$ ) could exhibit a transition between a counter-gradient to a gradient behavior when moving along the normal to the mean front from reactants toward products.

\section{A Particular Configuration: The Murray's Equation}

The Murray's equation [16] is a good example of the convection-diffusion equation, of the type considered here, to demonstrate that the common viewpoint that at the leading edge it is the diffusion term which always drives the flame propagation is not universal. Such an equation is obtained as a particular case of (9) corresponding to $R=1$, $u=0, \mathscr{D}=1$, and $\bar{w}=\widetilde{c}(1-\widetilde{c})$. In such a case, we have $f_{B}=1, f^{\prime}(0)=\left|f^{\prime}(1)\right|=1, \Omega^{\prime}(0)=\left|\Omega^{\prime}(1)\right|=1$, and $\beta=\alpha$. We note that formally though, $R=1$ corresponds to $\tau=0$ leading to $N_{B}=0$. But we will consider here $N_{B}$ like a parameter not linked to $\tau$. In such a situation, by denoting $K=2 \widehat{N}_{B}$ and $c_{w}=\Lambda+\widehat{N}_{B}$, (11) reduced to that analyzed by Murray [16] to determine the wave speed $c_{w}$, namely,

$$
\frac{d^{2} \tilde{c}}{d x^{2}}-\left(c_{w}-K \tilde{c}\right) \frac{d \tilde{c}}{d x}+\widetilde{c}(1-\tilde{c})=0 .
$$

Murray [16] showed that travelling wave solutions exist for all $c_{w} \geq c_{w}(K)$ where

$$
c_{w}(K)= \begin{cases}2 & \text { if } K<2, \\ \frac{k}{2}+\frac{2}{k} & \text { if } K \geqslant 2,\end{cases}
$$

or equivalently with our notations

$$
\Lambda= \begin{cases}2-\hat{N}_{B} & \text { if } \hat{N}_{B}<1, \\ \frac{1}{\hat{N}_{B}} & \text { if } \hat{N}_{B} \geqslant 1 .\end{cases}
$$

Thus, it appears that in that particular case, for $\hat{N}_{B} \geqslant 1$ and in the zone $\tilde{c} \rightarrow 0$, it is the counter-gradient transport that prevails (see the domain CG/G in Figures 3 and 4).

\section{Concluding Remark}

A methodology that permits to study the domain of the tubulent flame velocities for a steady regime of a turbulent premixed flame propagation has been presented. It has been shown how the introduction of the parameter $f_{B}$ (defined by 32 ) that combines both the counter-gradient flux and the mean reaction rate behavior at both edges of the flame can be used to determine the domain where a steady regime of propagation may exist for any combination of scalar fluxes that dominate at the flame edges. It is worth noticing though that the present analysis of the sole velocity domain does not prove the existence/unicity of the steady solutions. Consequently, future work will concentrate on the use of numerical simulations to study these questions.

\section{Acknowledgments}

The support of the first author by ONERA as well as the support of the second author by CNRS are greatly acknowledged.

\section{References}

[1] A. Kolmogorov, I. Petrovskii, and N. Piskounov, "Study of the diffusion equation with growth of the quantity of matter and its application to a biology problem," in Dynamics of Curved Fronts, P. Pelcé, Ed., pp. 105-130, Academic Press, Boston, Mass, USA, 1988.

[2] J. B. Moss, "Simultaneous measurements of concentration and velocity in an open pre-mixed flame," Combustion Science and Technology, vol. 22, no. 3-4, pp. 119-129, 1980.

[3] R. K. Cheng and I. G. Shepherd, "The influence of burner geometry on premixed turbulent flame propagation," Combustion and Flame, vol. 85, no. 1-2, pp. 7-26, 1991.

[4] G. Troiani, M. Marrocco, S. Giammartini, and C. M. Casciola, "Counter-gradient transport in the combustion of a premixed $\mathrm{CH} /$ air annular jet by combined PIV/OH-LIF," Combustion and Flame, vol. 156, no. 3, pp. 608-620, 2009.

[5] L. Zimmer, S. Tachibana, and K. Suzuki, "Mesures des vitesses conditionnées en flamme prémélangée," in Proceedings of the 9th Congrès Francophone de Vélocimétrie Laser, pp. A.1.1A.1.8, 2004.

[6] D. Veynante, A. Trouvé, K. N. C. Bray, and T. Mantel, "Gradient and counter-gradient scalar transport in turbulent premixed flames," Journal of Fluid Mechanics, vol. 332, pp. 263-293, 1997.

[7] S. Nishiki, DNS and modeling of turbulent premixed combustion, Ph.D. thesis, Nagoya Institute of Technology, Japan, 2003.

[8] R. Hauguel, "Flamme en V turbulente: simulation numérique directe et modélisation de la combustion turbulente prémélangée," Ph.D. thesis, Insa de Rouen, France, 2003.

[9] E. Lee and K. Y. Huh, "Zone conditional modeling of premixed turbulent flames at a high Damköhler number," Combustion and Flame, vol. 138, no. 3, pp. 211-224, 2004.

[10] A. N. Lipatnikov and J. Chomiak, "Effects of premixed flames on turbulence and turbulent scalar transport," Progress in Energy and Combustion Science, vol. 36, no. 1, pp. 1-102, 2010.

[11] V. L. Zimont and F. Biagioli, "Gradient, counter-gradient transport and their transition in turbulent premixed flames," Combustion Theory and Modelling, vol. 6, no. 1, pp. 79-101, 2002.

[12] A. N. Lipatnikov and J. Chomiak, "A study of the effects of pressure-driven transport on developing tubulent flame structure and propagation," Combustion Theory and Modelling, vol. 8, no. 2, pp. 211-225, 2004.

[13] C. Corvellec, P. Bruel, and V. A. Sabel'nikov, "Turbulent premixed flames in the flamelet regime: Burning velocity spectral properties in the presence of countergradient diffusion," Combustion and Flame, vol. 120, no. 4, pp. 585-588, 2000.

[14] D. Bradley, P. H. Gaskell, and X. J. Gu, "Application of a Reynolds stress, stretched flamelet, mathematical model to computations of turbulent burning velocities and comparison with experiments," Combustion and Flame, vol. 96, no. 3, pp. 221-248, 1994.

[15] R. K. Cheng, "Velocity and scalar characteristics of premixed turbulent flames stabilized by weak swirl," Combustion and Flame, vol. 101, no. 1-2, pp. 1-14, 1995.

[16] J. D. Murray, Mathematical Biology, Springer, Berlin, Germany, 1994. 

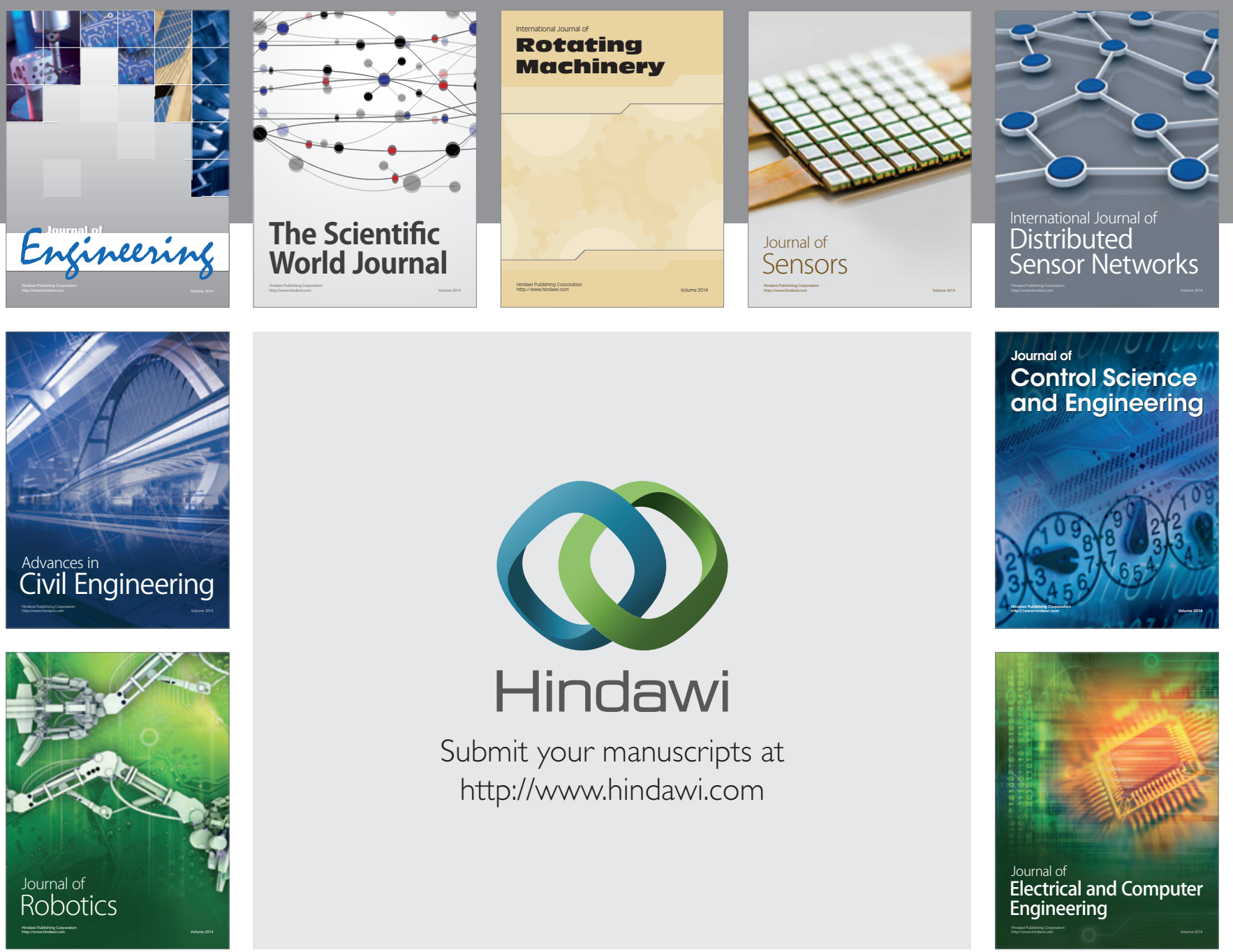

Submit your manuscripts at

http://www.hindawi.com
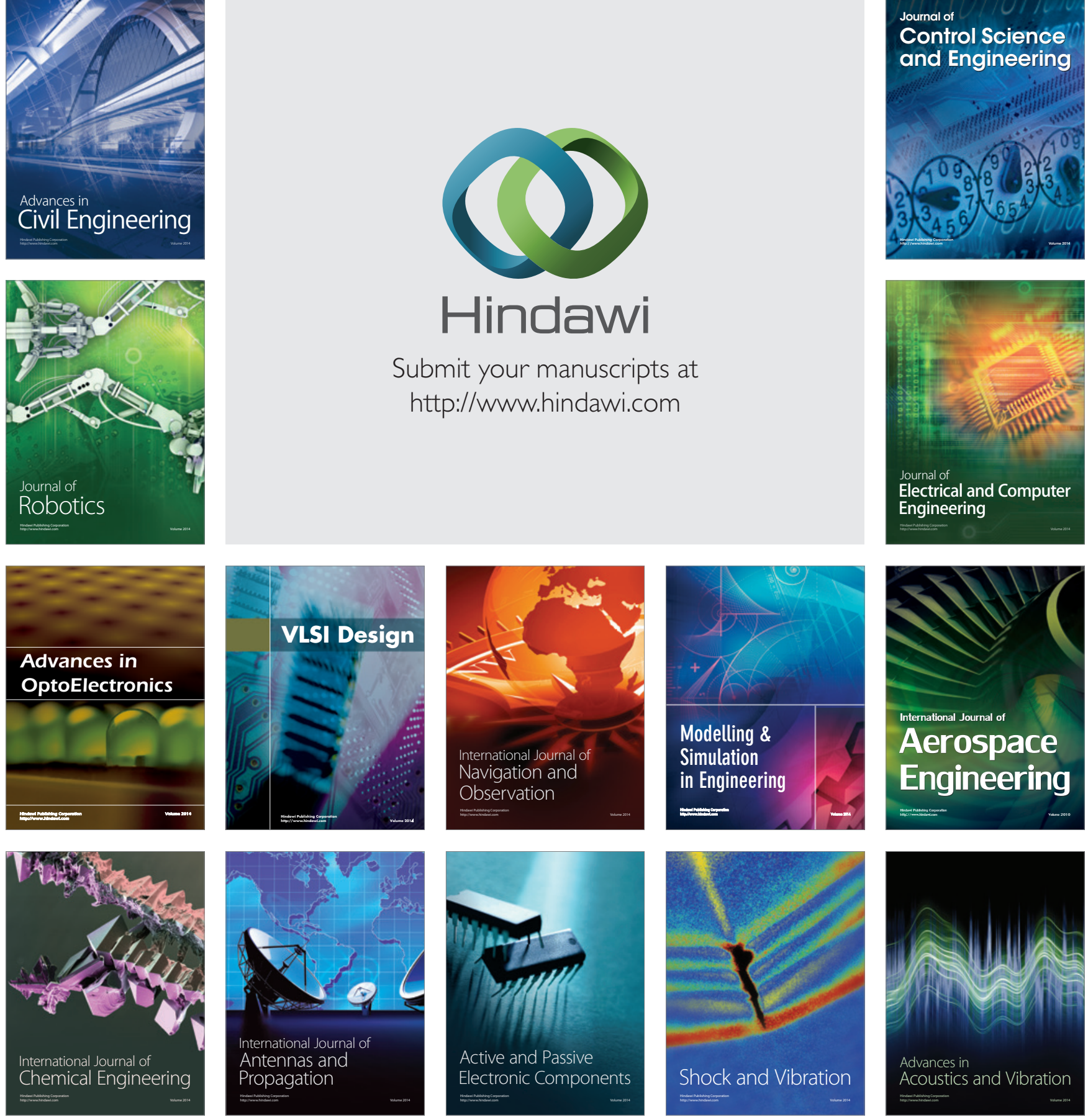